\title{
Ion-Specific Oil Repellency of Polyelectrolyte Multilayers in Water: Molecular Insights into the Hydrophilicity of Charged Surfaces**
}

\author{
Xiaokong Liu, Chuan Leng, Li Yu, Ke He, Lauren Joan Brown, Zhan Chen, Jinhan Cho, and \\ Dayang Wang*
}

\begin{abstract}
Surface wetting on polyelectrolyte multilayers (PEMs), prepared by alternating deposition of polydiallyldimethylammonium chloride (PDDA) and poly(styrene sulfonate) (PSS), was investigated mainly in water-solid-oil systems. The surface-wetting behavior of as-prepared PEMs was well correlated to the molecular structures of the uncompensated ionic groups on the PEMs as revealed by sum frequency generation vibrational and $X$-ray photoelectron spectroscopies. The orientation change of the benzenesulfonate groups on the PSS-capped surfaces causes poor water wetting in oil or air and negligible oil wetting in water, while the orientation change of the quaternized pyrrolidine rings on the PDDA-capped surfaces hardly affects their wetting behavior. The underwater oil repellency of PSS-capped PEMs was successfully harnessed to manufacture highly efficient filters for oil-water separation at high flux.
\end{abstract}

$A$ study of water wetting on a charged surface will not only advance our knowledge of many peculiar surface functions, such as lubrication, anti-fouling, and self-cleaning, which are encountered in biological systems, but also enhance our ability to translate these surface functions into innovative technical applications. ${ }^{[1]}$ In general, charged surfaces are easily wetted by water, which is a macroscopic expression of strong hydration of the surface ionic groups. For a solid surface, air drying is obviously inevitable in practice, even if is not required technically. Upon contact with air, charged surfaces, like many other polar surfaces with high surface free energy, must orient the ionic groups inwards to lower the surface free energy, thus resulting in a noticeable increase in surface hydrophobicity. As such, wetting charged surfaces with water, characterized by the water contact angle in air $\left(\theta_{\mathrm{w} /}\right.$ a), has little dependence on the surface ionic group, though the interaction of ions or ionic groups with water (hydration)

[*] Dr. X. Liu, L. Yu, K. He, L. J. Brown, Prof. Dr. D. Wang Ian Wark Research Institute, University of South Australia Mawson Lakes, SA 5095 (Australia)

E-mail: dayang.wang@unisa.edu.au

C. Leng, Prof. Dr. Z. Chen

Department of Chemistry, University of Michigan

Ann Arbor, MI 48109 (USA)

Prof. Dr. J. Cho

Department of Chemical and Biological Engineering, Korea University, Seoul, 136-701 (Korea)

[**] Australian Research Council (DP120102959) and US Office of Nava Research (N00014-12-1-0452) are acknowledged for financial support.

Supporting information for this article is available on the WWW under http://dx.doi.org/10.1002/anie.201411992. is known to be ion-specific. ${ }^{[2]}$ In contrast, the present work demonstrates a prominent dependence of the surface hydrophilicity of polyelectrolyte multilayers (PEMs) on the molecular nature of the uncompensated ionic groups presented at the surface.

Herein we study surface wetting of the PEMs, which are obtained by layer-by-layer (LbL) deposition of polydiallyldimethylammonium chloride (PDDA) and poly(styrene sulfonate) (PSS), mainly in water-solid-oil systems. Our results demonstrate that the surface hydrophilicity of the resulting PEMs is correlated primarily to the molecular configuration rather than hydration strength of the uncompensated ionic groups on the surfaces. According to a newly developed theory on the correlation between the water contact angle and surface polarity, ${ }^{[3]}$ the surface hydrophilicity of PEMs should be determined by the surface charge density, which can be defined by the surface area occupied by the surface ionic groups. For PSS-capped surfaces, the uncompensated benzenesulfonate $\left(\mathrm{BS}^{-}\right)$groups on the surfaces comprise rigid and hydrophobic phenyl $(\mathrm{Ph})$ moieties and hydrophilic, anionic sulfonate $\left(\mathrm{SO}_{3}{ }^{-}\right)$moieties, which are well separated in space (see Scheme S1 in the Supporting Information). Our spectroscopic results show that in water, the surface $\mathrm{BS}^{-}$groups orient their $\mathrm{Ph}$ moieties in an orderly and upright position out of the surface of the plane. This orientation maximizes surface charge density and transforms the surface hydration of the $\mathrm{SO}_{3}{ }^{-}$moieties into oil repellency with an oil contact angle in water $\left(\theta_{\mathrm{o} / \mathrm{w}}\right)$ of greater than $165^{\circ}$. Upon contact with air or oil, the $\mathrm{Ph}$ moieties are randomly distributed and orient parallel to the surface plane, and thus significantly reduces the surface charge density. As a result, the PSS-capped surfaces become poorly wetted by water, as evidenced by the water contact angle in oil $\left(\theta_{\mathrm{w} / \mathrm{o}}\right)$ of greater than $90^{\circ}$. In contrast, the uncompensated quaternary amine $\left(\mathrm{QA}^{+}\right)$groups on PDDAcapped surfaces have their cationic $\left(\mathrm{N}^{+}\right)$center enveloped by four alkyl moieties in each pyrrolidine ring (see Scheme S1). Owing to this configuration, the surface charge density and surface hydrophilicity of the PDDA capped surfaces are insensitive to the orientation of the quaternized pyrrolidine ring. They remain well wetted by water in air and in oil $\left(\theta_{\mathrm{w} / \mathrm{o}}=\right.$ $42^{\circ}$ ), though the weak hydration of the surface $\mathrm{QA}^{+}$groups cannot effectively prevent oil adhesion in water $\left(\theta_{\mathrm{o} / \mathrm{w}}=\mathrm{ca}\right.$. $\left.133^{\circ}\right)$. Thus, our results demonstrate a pronounced dependence of the wetting behavior of charged surfaces on the molecular nature and geometric configuration of the surface ionic groups. This dependence will also provide a better model to describe how ionic species interact with water, air, and oil at interfaces where a marked conformational change is essential for adsorption and translocation. 
Surface wetting is conventionally studied in water-solidair systems, and our study mainly focused on water-solid-oil systems because of the academic importance ${ }^{[4]}$ and strong relevance in industry. ${ }^{[5]}$ In this context, oil/water separation is an important technical application. To date, the prevailing strategy is to make hydrophilic nanostructures on the surfaces of separation membranes, inspired by the oil-repellent skins of fish and other aquatic organisms. ${ }^{[6]}$ Such surface nanostructures are designed for water trapping, and can effectively offset the potential surface hydrophilicity loss because of their air-drying-induced surface reconstruction, thus ensuring underwater surface superoleophobicity. Here we discover that upon immersion into water, PSS-capped PEMs exhibit excellent oil-repellency regardless of aging treatment in air. This repellency has encouraged us to coat conventional steel meshes with PSS-capped PEMs, thus enabling highly efficient separation of water from oil/water mixtures at high flux, and opening promising prospects in the oil-water separation industry.

To date, a large variety of polyelectrolytes have been used to produce PEMs by LbL electrostatic deposition. ${ }^{[7]}$ PDDA and PSS are the most commonly used, permanently charged polyelectrolytes. Here they were alternatingly deposited onto silicon wafers in the presence of $1.0 \mathrm{M} \mathrm{NaCl}$ and the resulting PEMs were denoted as (PDDA/PSS $)_{n}$ where " $n$ " represents the bilayer numbers. In this work, we mainly studied (PDDA/ PSS $)_{3.5}$ and (PDDA/PSS $)_{4.0}$ PEMs with a surface roughness of less than $3 \mathrm{~nm}$ (see Figure S1 in the Supporting Information), so the impact of the surface roughness on surface wetting was ignored. The surface compositions of as-prepared (PDDA/ PSS $)_{n}$ PEMs were examined by X-ray photoelectron spectroscopy (XPS; see Figure S2 and Table S1 in the Supporting Information). Figure $1 \mathrm{a}$ shows that the $\mathrm{N}$ to $\mathrm{S}$ atomic ratio is alternatingly switched from about. 0.7 for PSS-capped PEMs to 1.7-2.0 for PDDA-capped PEMs. The type of the counterions present on the PEMs, determined by XPS, is indicative of the charge balance during alternating deposition of PDDA and PSS. ${ }^{[8]}$ Figure $1 \mathrm{~b}$ shows that the counterions of the $\mathrm{QA}^{+}$ groups, $\mathrm{Cl}^{-}$ions, are detected exclusively on the PDDAcapped surfaces while the counterions of the $\mathrm{BS}^{-}$groups, $\mathrm{Na}^{+}$ ions, are exclusively detected on the PSS-capped surfaces, thus indicating surface charge overcompensation during alternating adsorption of PDDA and PSS. This observation, however, is different from the recent report of Schlenoff et al., in which the surface charge overcompensation was observed only for PDDA adsorption. ${ }^{[8]}$ According to XPS measurements of (PDDA/PSS) ${ }_{n}$ PEMs obtained at different $\mathrm{NaCl}$ concentrations (see Figure S3 and Table S2 in the Supporting Information), ${ }^{[9]}$ we observe that the atomic concentration of $\mathrm{Na}^{+}$ions detected on the PSS-capped surfaces decreases from $3.5 \%$ to $1.3 \%$ with the $\mathrm{NaCl}$ concentrations used for LbL deposition decreasing from 1.0 to $0.5 \mathrm{M}$, and becomes undetectable when $\mathrm{NaCl}$ concentrations further reduce to 0.25 and $0.1 \mathrm{M}$ (Figure $1 \mathrm{c}$ ). In contrast, $\mathrm{Cl}^{-}$ions are clearly detected on all the PDDA-capped surfaces obtained at different $\mathrm{NaCl}$ concentrations (Figure 1d). Schlenoff et al. observed overcompensation upon PSS deposition at $\mathrm{NaCl}$ concentrations of greater than $1.5 \mathrm{M}$, and was attributed to $\mathrm{NaCl}$-induced $\mathrm{PEM}$ plasticization. ${ }^{[8]}$ While this plasticization could account for our results, here PSS overcompensation occurred at $0.5 \mathrm{M} \mathrm{NaCl}$. A detailed study of the $\mathrm{NaCl}$ concentration effect on the amount of counterions detected on (PDDA/PSS $)_{n}$ PEMs, is underway.

From the XPS data, we estimated that $25-35 \%$ of the $\mathrm{QA}^{+}$groups and $70-80 \%$ of the $\mathrm{BS}^{-}$groups are uncompensated for on the (PDDA/PSS) 3.5 and (PDDA/PSS $)_{4}$ PEM surfaces obtained in $1.0 \mathrm{M} \mathrm{NaCl}$. Figure $2 \mathrm{a}$ shows that the resulting PEMs can be readily wetted by water in air, with a $\theta_{\mathrm{w} / \mathrm{a}}$ value of $15-20^{\circ}$, and is a result of the effective hydration of the surface ionic groups. After 72 hours of aging at $60^{\circ} \mathrm{C}$ in air, the $\theta_{\mathrm{w} / \mathrm{a}}$ values increased to about $34^{\circ}$ and $51^{\circ}$ for (PDDA/ PSS $)_{3.5}$ and (PDDA/PSS $)_{4}$ PEMs, respectively, thus suggesting that the surface hydrophilicity of the PSS capping is weaker and more vulnerable to air aging than the PDDA capping. When oil is utilized instead of air to challenge water wetting on the resulting PEMs, as shown in Figure $2 b$, the PDDAcapped surfaces remain easily wetted by water $\left(\theta_{\mathrm{w} / \mathrm{o}} \approx 42^{\circ}\right)$, while water wetting on the PSS-capped surfaces becomes noticeably poorer, with $\theta_{\mathrm{w} / \mathrm{o}}$ decreasing from $110^{\circ}$ to an equilibrium value of $97^{\circ}$ over time. These results are inconsistent with the hydration strength of surface ionic groups. As suggested in the literature, ${ }^{[2 \mathrm{~d}]}$ the $\mathrm{BS}^{-}$groups (i.e., their $\mathrm{SO}_{3}{ }^{-}$ moieties) on PSS-capped surfaces are expected to be more strongly hydrated than the $\mathrm{QA}^{+}$groups on PDDA-capped
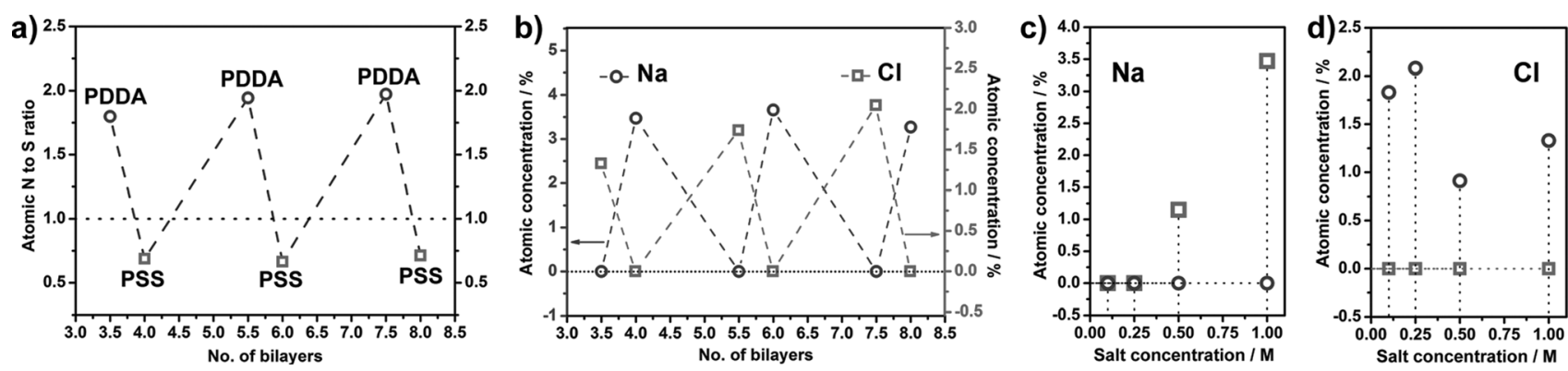

Figure 1. a) Plot of the $\mathrm{N}$ to $\mathrm{S}$ atomic ratios on (PDDA/PSS) $)_{n} \mathrm{PEMs}$, prepared by LbL deposition in $1.0 \mathrm{M} \mathrm{NaCl}$, versus the bilayer number. b) Plot of the atomic concentration of $\mathrm{Na}$ (circles) and $\mathrm{Cl}$ (squares) on (PDDA/PSS) ${ }_{n}$ PEMs, prepared by LbL deposition in $1.0 \mathrm{M} \mathrm{NaCl}$, versus the bilayer number. Plot of the atomic concentration of $\mathrm{Na}$ (c) and $\mathrm{Cl}$ (d) on PDDA- (circles) and PSS-capped (squares) surfaces versus the $\mathrm{NaCl}$ concentration used for LbL growth of (PDDA/PSS) ${ }_{n}$ PEMs. In Figure $c$ and d, (PDDA/PSS) ${ }_{3.5}$ and (PDDA/PSS) 4.0 PEMs, obtained in 1.0 NaCl, are compared with the PDDA-capped and PSS-capped PEMs, obtained in dilute $\mathrm{NaCl}$, with similar thickness and roughness. ${ }^{[9]}$ All the data are obtained from XPS measurements. 

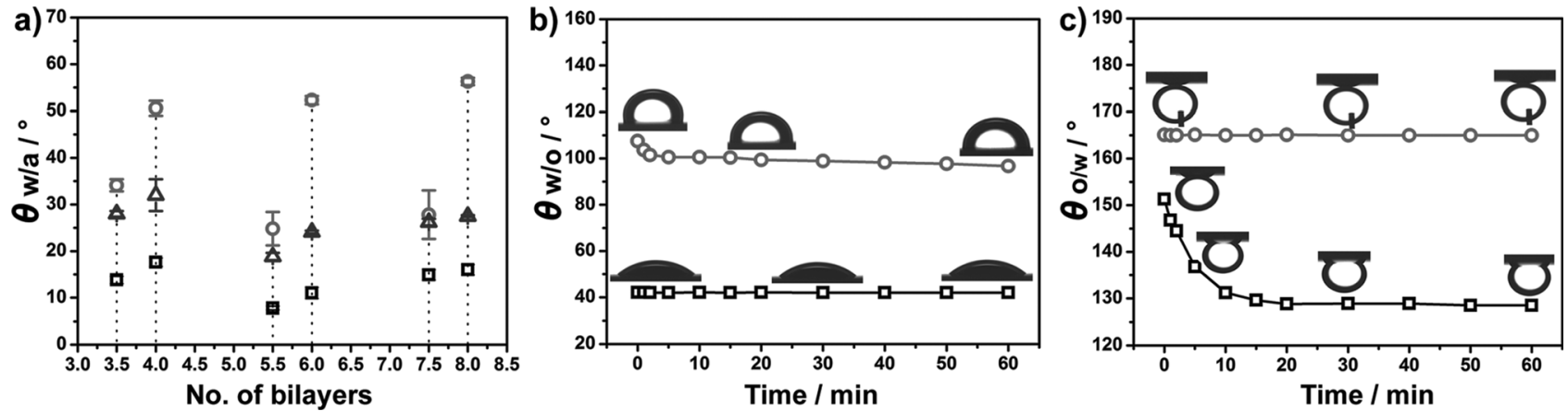

Figure 2. a) Plot of the $\theta_{\mathrm{w} / \mathrm{a}}$ values of (PDDA/PSS) PEMs, which were freshly prepared (squares), aged for 72 hours at room temperature (triangles), and at $60^{\circ} \mathrm{C}$ (circles), versus the bilayer number. b,c) Temporal evolution profiles of the $\theta_{\mathrm{w} / \mathrm{o}}$ (b) and $\theta_{\mathrm{o} / \mathrm{w}}$ (c) values of (PDDA/PSS) 3.5 (squares) and (PDDA/PSS) 4.0 (circles) PEMs. The PEMs are prepared by LbL deposition in $1.0 \mathrm{M} \mathrm{NaCl}$.

surfaces. Obviously, this inconsistency cannot be simply reconciled by surface reconstruction of the surface ionic groups upon contact with air or oil.

We also studied oil wetting on (PDDA/PSS) ${ }_{n}$ PEMs. Oil can completely wet the (PDDA/PSS) $)_{n}$ PEMs in air, with the oil contact angle in air $\left(\theta_{\mathrm{o} / \mathrm{a}}\right)$ at less than $5^{\circ}$. Figure $2 \mathrm{c}$ shows oil wetting on (PDDA/PSS $)_{n}$ PEMs in water. When the PEMs are brought in contact with a pendant droplet of hexadecane $(2 \mu \mathrm{L})$ in water, both (PDDA/PSS $)_{3.5}$ and (PDDA/PSS $)_{4}$ PEMs initially exhibit a $\theta_{\mathrm{o} / \mathrm{w}}$ value above $150^{\circ}$. With the prolonged contact time, however, the $\theta_{\mathrm{o} / \mathrm{w}}$ value of the PDDAcapped surfaces gradually drops from $152^{\circ}$ to $133^{\circ}$ within 20 minutes while that of the PSS-caped surfaces remains as high as $165^{\circ}$ with no detectable change. When the PEMs were removed from the hexadecane droplet, the oil droplets stayed on the PDDA-capped surfaces (see Figure S4a and Movie S1 in the Supporting Information), whereas complete oil detachment was observed on the PSS-capped surfaces, even after the surfaces were kept in tight contact with the oil droplets for 12 hours (see Figure S4b and Movie S2 in the Supporting Information). This excellent oil repellency, however, was not found on the PSS-capped PEMs made by LbL deposition in 0.25 and $0.10 \mathrm{M} \mathrm{NaCl}$; the oil droplet stably adhered to the surfaces despite the large $\theta_{\mathrm{o} / \mathrm{w}}$ value (ca. $155^{\circ}$; see Figure S5 in the Supporting Information). Since complete compensation occurs upon PSS deposition in dilute $\mathrm{NaCl}$ solutions (Figure $1 \mathrm{c}$ ), we believe that the above observed oil repellency of the PSS-capped PEMs prepared in $1.0 \mathrm{M} \mathrm{NaCl}$ arises from strong hydration of uncompensated $\mathrm{BS}^{-}$groups on the surfaces. Distinct from water wetting in air or oil, oil wetting in water is clearly consistent with the reported hydration strength of the ionic groups on the resulting PEMs. ${ }^{[2 \mathrm{~d}]}$

Based on the Young equation, the $\theta_{\mathrm{w} / \mathrm{a}}, \theta_{\mathrm{o} / \mathrm{a}}, \theta_{\mathrm{w} / \mathrm{o}}$, and $\theta_{\mathrm{o} / \mathrm{w}}$ of a solid surface are correlated by:

$$
\begin{aligned}
& \cos \theta_{\mathrm{o} / \mathrm{w}}=\left(\gamma_{\mathrm{o} / \mathrm{a}} \cos \theta_{\mathrm{o} / \mathrm{a}}-\gamma_{\mathrm{w} / \mathrm{a}} \cos \theta_{\mathrm{w} / \mathrm{a}}\right) / \gamma_{\mathrm{o} / \mathrm{w}} \\
& \theta_{\mathrm{o} / \mathrm{w}}+\theta_{\mathrm{w} / \mathrm{o}}=180^{\circ}
\end{aligned}
$$

where $\gamma_{\mathrm{o} / \mathrm{a}}, \gamma_{\mathrm{w} / \mathrm{a}}$, and $\gamma_{\mathrm{o} / \mathrm{w}}$ are the oil/air, water/air, and oil/water interfacial tensions, respectively. To simplify the calculation, we approximated that $\theta_{\mathrm{o} / \mathrm{a}}=5^{\circ}$ (for hexadecane) and $\theta_{\mathrm{w} / \mathrm{a}}=30^{\circ}$ for both (PDDA/PSS) $)_{3.5}$ and (PDDA/PSS $)_{4}$ PEMs, values which are reasonable for the PEMs obtained after 72 hours of aging in air (Figure 2a). According to Equations (1) and (2), $\theta_{\mathrm{o} / \mathrm{w}}$ and $\theta_{\mathrm{w} / \mathrm{o}}$ are calculated to be $135^{\circ}$ and $45^{\circ}$, respectively. These theoretical values are similar to the experimentally measured data of the (PDDA/PSS $)_{3.5} \operatorname{PEM}\left(\theta_{\mathrm{o} / \mathrm{w}}=133^{\circ}\right.$ and $\left.\theta_{\mathrm{w} / \mathrm{o}}=42^{\circ}\right)$, but considerably smaller than those of the $(\mathrm{PDDA} / \mathrm{PSS})_{4} \operatorname{PEM}\left(\theta_{\mathrm{o} / \mathrm{w}}=165^{\circ}\right.$ and $\left.\theta_{\mathrm{w} / \mathrm{o}}=97^{\circ}\right) ; \theta_{\mathrm{o} / \mathrm{w}}+\theta_{\mathrm{w} / \mathrm{o}}=$ $262^{\circ}\left(>180^{\circ}\right) .^{[10]}$

To reveal the molecular rationale for their complicated wetting behavior, the surface molecular structures of the $(\text { PDDA/PSS })_{3.5}$ and (PDDA/PSS) ${ }_{4}$ PEMs, obtained by LbL deposition in $1.0 \mathrm{M} \mathrm{NaCl}$, were investigated by sum frequency generation vibrational spectroscopy (SFG; Figure 3). SFG is an intrinsically surface-specific method which provides information about molecular conformation and orientation at various interfaces. ${ }^{[1]}$ Figure $3 \mathrm{a}$ shows that in water the uncompensated $\mathrm{QA}^{+}$groups on the (PDDA/PSS) ${ }_{3.5}$ PEMs exhibit fairly strong $\mathrm{C}-\mathrm{N}^{+}$stretching vibrational signal, thus indicating the surface $\mathrm{QA}^{+}$groups preferentially orient perpendicular to the surface plane (Figure $4 \mathrm{~b}$ ). The strong $\mathrm{CH}_{3}$ stretching signal in water suggests that each $\mathrm{QA}^{+}$group has two $\mathrm{CH}_{3}$ moieties normal to the surface in direct contact with water (Figure $4 \mathrm{~b}$ ). The $\mathrm{CH}_{2}$ stretching signal is also strong in water (compared to that in air), though weaker than the $\mathrm{CH}_{3}$ one, thus indicating that the two $\mathrm{CH}_{2}$ moieties of the pyrrolidine ring are also ordered on the surface and directly in contact with water. ${ }^{[12]}$ This orientational configuration agrees with the molecular structures of the surface $\mathrm{QA}^{+}$groups where the $\mathrm{N}^{+}$center isotropically enveloped by two $\mathrm{CH}_{3}$ and two $\mathrm{CH}_{2}$ moieties in each pyrrolidine ring (see Scheme S1), and accounts for the wetting behavior of the PDDA-capped surface in water. This alkyl envelopment greatly lowers the hydration effectiveness of the $\mathrm{N}^{+}$center and, at the same time, facilitates oil adhesion on the PDDA-capped surface in water. When the PDDA-capped surface is exposed to air, the $\mathrm{CH}_{3}$ stretching signal becomes weaker than that of $\mathrm{CH}_{2}$ (Figure $3 \mathrm{a}$ ), thus indicating that the surface $\mathrm{QA}^{+}$groups orient parallel to the surface plane with two $\mathrm{CH}_{2}$ moieties of the pyrrolidine ring protruding more from the surface plane than the two $\mathrm{CH}_{3}$ moieties (Figure $4 \mathrm{a}$ ). This orientation change may just slightly change the surface area occupied by each $\mathrm{QA}^{+}$group and in turn the surface charge density on the PDDA capped surface, because quaternized pyrrolidine rings 


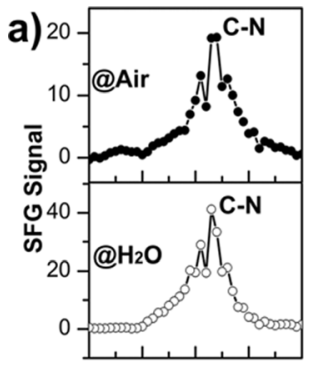

13501400145015001550 Wavenumber $/ \mathrm{cm}^{-1}$

b)

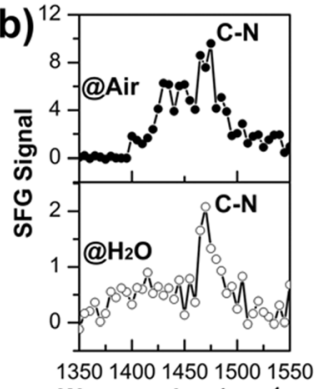

Wavenumber $/ \mathrm{cm}^{-1}$
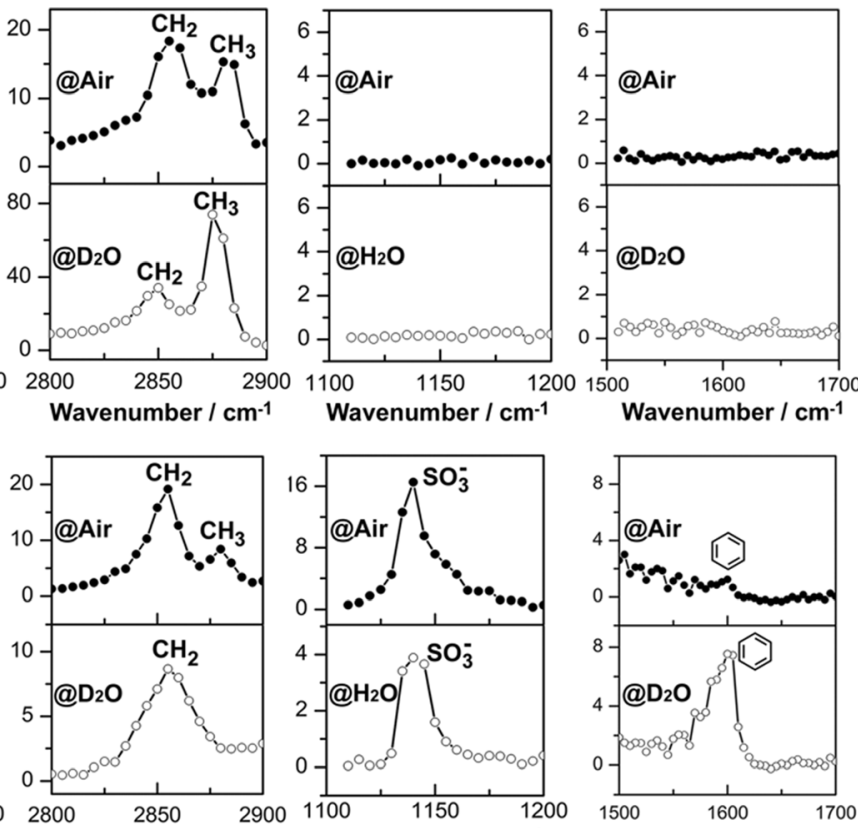

Wavenumber $/ \mathrm{cm}^{-1}$

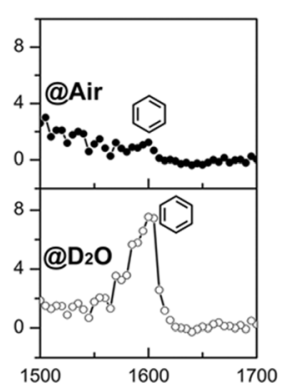

Wavenumber $/ \mathrm{cm}^{-1}$

Figure 3. SFG spectra of (PDDA/PSS) ${ }_{3.5}$ (a) and (PDDA/PSS) ( .0 (b) PEMs measured in air (upper panel) and water (lower panel). The PEMs were produced by LbL deposition in $1.0 \mathrm{NaCl}$. Deuterated water is used instead of water to distinctly reveal the vibration signal of alkyl and phenyl moieties.

ordering in water, individual surface $\mathrm{BS}^{-}$groups on the PSS-capped surfaces will occupy minimal surface area (Figure 4b), thus maximizing the surface charge density and transforming the strong surface hydration of the $\mathrm{SO}_{3}{ }^{-}$moieties into strong oil repellency in water. In air, however, the $\mathrm{Ph}$ moieties randomly orient parallel to the surface plane, so the surface area occupied by each surface $\mathrm{BS}^{-}$group is maximized (Figure $4 \mathrm{a}$ ), thus significantly reducing the surface charge density and in turn the surface hydrophilicity. This arrangement explains the poor water wetting on PSS-capped surfaces in oil/air.

Whereas neither $\mathrm{SO}_{3}{ }^{-}$nor $\mathrm{Ph}$ moieties of $\mathrm{BS}^{-}$groups are visible in the SFG spectra collected from the (PDDA/PSS) ${ }_{3.5}$ PEM, the $\mathrm{C}-\mathrm{N}^{+}$and $\mathrm{CH}_{3}$ signals of the $\mathrm{QA}^{+}$groups were detected in the SFG spectra of the $(\mathrm{PDDA} / \mathrm{PSS})_{4} \quad$ PEM (Figure $3 \mathrm{~b}$ ). This detection implies that despite the surface charge overcompensa-

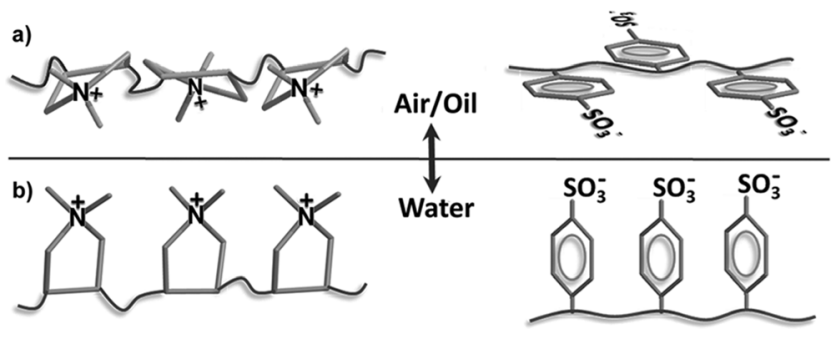

Figure 4. Schematic illustration of the orientational configuration of the uncompensated $\mathrm{QA}^{+}$groups on (PDDA/PSS) ${ }_{3.5}$ PEMs and the uncompensated $\mathrm{BS}^{-}$groups on (PDDA/PSS) ${ }_{4.0}$ PEMs in response to the surrounding environmental change.

are inserted as bulky repeating unites in the PDDA backbone and the $\mathrm{N}^{+}$center of each ring is enveloped by two $\mathrm{CH}_{3}$ and two $\mathrm{CH}_{2}$ moieties. The distinct $\mathrm{C}-\mathrm{N}^{+}$stretching signal in air, albeit weaker than that in water, indicates that the surface $\mathrm{QA}^{+}$groups point the $\mathrm{N}^{+}$centers towards air, and facilitates the surface hydration. Hence the surface charge density on the PDDA-capped surfaces is little altered and, therefore, the water wetting remains effective regardless of the orientation of the surface $\mathrm{QA}^{+}$groups in response to the environmental change.

Figure $3 \mathrm{~b}$ shows that the $\mathrm{SO}_{3}{ }^{-}$moieties of the uncompensated $\mathrm{BS}^{-}$groups on the (PDDA/PSS) ${ }_{4} \mathrm{PEMs}$ are present both in air and water, but the aromatic $\mathrm{C}=\mathrm{C}$ stretching signal of the $\mathrm{Ph}$ moieties appears only in water, thus indicating the different orientation and ordering of the $\mathrm{Ph}$ moieties in air and water. Since surface $\mathrm{BS}^{-}$groups have the $\mathrm{SO}_{3}{ }^{-}$and $\mathrm{Ph}$ moieties placed side by side (see Scheme S1), and when their $\mathrm{Ph}$ moieties orient perpendicular to the surface plane with tion upon PSS adsorption in $1.0 \mathrm{M} \mathrm{NaCl}$, a small number of the $\mathrm{QA}^{+}$groups from the PDDA layer beneath, as evidenced by their weaker intensities compared to those observed on the PDDA-capped PEM surface, remain exposed to the environment, along with the $\mathrm{BS}^{-}$groups of the PSS capping. This finding inspired us to focus on understanding the internal structure of (PDDA/PSS) ${ }_{n}$ PEMs, as it is still under debate. Figure $3 \mathrm{~b}$ indicates the absence of the $\mathrm{CH}_{3}$ stretching vibration signal of $\mathrm{QA}^{+}$groups in water, and it is reasonable when the surface $\mathrm{BS}^{-}$groups stand up on the surfaces in water, and is clearly beneficial for the PSS-capped surfaces to keep hydrated and repel oil in water.

According to literature ${ }^{[13]}$ counterions are expected to be strongly bound with the surface ionic groups on as-prepared (PDDA/PSS $)_{n}$ PEMs through electrostatic interactions and in turn affect the surface wetting behavior. To study the counterion effect, the counterions of the surface $\mathrm{QA}^{+}$ groups were changed from $\mathrm{Cl}^{-}$to $\mathrm{Br}^{-}$ions by adsorption of the outmost PDDA layer in $1.0 \mathrm{M} \mathrm{NaBr}$ and the counterions of the surface $\mathrm{BS}^{-}$groups from $\mathrm{Na}^{+}$to $\mathrm{K}^{+}$ions by adsorption of the outmost PSS layer in 1.0 KCl (see Figure S6 and Table S3 in the Supporting Information).$^{[14,15]}$ The counterion nature is found to noticeably affect $\theta_{\mathrm{w} / \mathrm{a}}$ values of the resulting PEMs according to the Hofmeister series ${ }^{[2 a, b]}$ (see Figure S7a in the Supporting Information), while its impact on their $\theta_{\mathrm{w} / \mathrm{o}}$ value is clearly dependent on the surface ionic group nature (see Figure S7b). With the counterion polarizibility increasing from $\mathrm{Cl}^{-}$to $\mathrm{Br}^{-}$ions and from $\mathrm{Na}^{+}$to $\mathrm{K}^{+}$ions, ${ }^{[2 a, b]}$ the $\theta_{\mathrm{w} /}$ o value noticeably increases for the PDDA capping while it is little changed for the PSS capping. Further, the $\theta_{\mathrm{o} / \mathrm{w}}$ value of the PEMs is hardly affected by the counterion nature (see Figure S7c). Taken together, water (de-)wetting on (PDDA/ 
PSS $)_{n}$ PEMs ought to be more significantly correlated with the surface ionic group nature.

We also capped (PDDA/PSS) ${ }_{n}$ PEMs with poly(allyl amine) (PAH) and poly(acrylic acid) (PAA) in the presence of $1.0 \mathrm{M} \mathrm{NaCl}^{[14]}$ Neither PAH nor PAA capping is oilrepellent (see Figure S7c), which implies that the PAH and PAA chains capped on the PEMs readily orient their hydrophobic moieties or backbones towards oil. This orientation is reasonable considering the small monomer volumes of $\mathrm{PAH}$ and PAA compared with those of PSS and PDDA (see Scheme S1). The PAH capping displays rather large $\theta_{\mathrm{w} / \mathrm{a}}$ and $\theta_{\mathrm{w} / \mathrm{o}}$ values (see Figure S7). This poor water wetting hints that the reconstruction of the surface allylamine groups in air or oil significantly reduces the surface charge density of the PAH capping, and is in line with the anisotropic configuration of the allylamine groups, similar to the $\mathrm{BS}^{-}$groups of PSS, in which their $\mathrm{CH} 2$ and $\mathrm{NH}_{3}^{+}$moieties are well separated. In contrast, the PAA capping is readily wetted by water with small $\theta_{\mathrm{w} / \mathrm{a}}$ and $\theta_{\mathrm{w} / \mathrm{o}}$ values, and is comparable to those of the PDDA capping (see Figure S7). This behavior can be assigned to the fact that the $\mathrm{COO}^{-}$groups of PAA, similar to the QA + groups of PDDA, are directly linked to the backbones so the orientation of the surface $\mathrm{COO}^{-}$group may just slightly change the surface charge density. Thus, these results further corroborate the strong correlation of the surface wetting behavior of PEMs with the surface ionic group nature.

After the surface reconstruction in air, hydrophilic surfaces are usually hard to restore their original hydrophilicity even by immersion in water. ${ }^{[16]}$ Because of the high flexibility of the PEMs obtained by LbL deposition, ${ }^{[15]}$ PSScapped PEMs were found to be capable of retaining the superior oil-repellency immediately after immersion in water, regardless of the post aging treatment (see Figure S8 in the Supporting Information). Encouraged by that, we coated stainless steel meshes with apertures of $25 \mu \mathrm{m}$ by (PDDA/ PSS $)_{n}$ PEMs through LbL deposition in $1.0 \mathrm{M} \mathrm{NaCl}$. Since the oil-repellency performance is independent on the layer number (see Figure S9 in the Supporting Information), smooth (PDDA/PSS $)_{4}$ PEMs were used for the mesh coating, which are thick enough to fully cover the mesh surface as evidenced by the disappearance of metal signals in XPS spectra (Figure $5 \mathrm{a}$ ). The resulting meshes allow easy filtration of water from an oil/water mixture at high water flux of about $610 \mathrm{~L} \mathrm{~m}^{-2} \mathrm{~s}^{-1}$ (Figure 5b and see Movie S3 in the Supporting Information) with high separation efficiency; oil is barely detected in the filtrated water (see Figure S10 in the Supporting Information). The surface roughness of the (PDDA/PSS) ${ }_{4}$ coating is less than $3 \mathrm{~nm}$ (see Figure S1b), and the notches and scratches present on the steel meshes are on the scale of tens to hundreds of micrometers (see Figure S11 in the Supporting Information). Thus, the contribution of the surface roughness to the high oil/water separation efficiency can be ignored. Since the (PDDA/ $\mathrm{PSS})_{4}$ coating were produced by $\mathrm{LbL}$ deposition in $1.0 \mathrm{M}$ $\mathrm{NaCl}$, its superior oil-repellency in water exhibited long term stability against acid, alkaline, and salt at high concentrations, and will be advantageous for technical applications such as oil-spill cleanup on seawater.
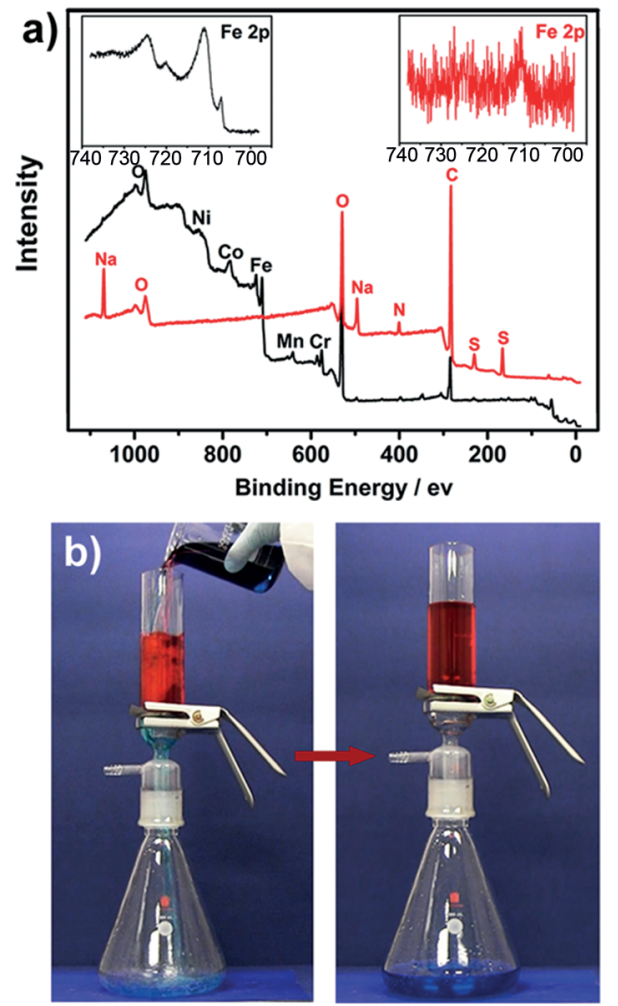

Figure 5. a) XPS wide scan spectra of a stainless steel mesh with (red) and without (black) (PDDA/PSS) ${ }_{4}$ PEMs coated atop by LbL deposition in $1.0 \mathrm{M} \mathrm{NaCl}$. The insets show the corresponding high-resolution spectra of $\mathrm{Fe}$ in the steel mesh. b) Photos shot during filtration of the mixtures of hexadecane, stained by Oil Red $O$, and water, stained by methylene blue, through a (PDDA/PSS) ${ }_{4}$-coated stainless steel mesh (left panel) and after the filtration (right panel), in which water is selectively filtered through the steel mesh while hexadecane is retained on the top of the mesh. The aperture of the steel mesh is $25 \mu \mathrm{m}$.

In summary, we demonstrate a prominent impact of the nature of the surface ionic group on the surface wetting behavior of (PDDA/PSS $)_{n}$ PEMs, and it hinges mainly on the configurational geometry of the uncompensated ionic groups on the surfaces. For PDDA-capped surfaces, the orientation of the surface $\mathrm{QA}^{+}$groups with isotropic configuration with respect to the surface plane hardly affect the surface hydrophilicity. For PSS-capped surfaces, the strong hydration of surface $\mathrm{BS}^{-}$groups with anisotropic configuration can be translated either into excellent oil-repellency in water (poor water de-wetting) or to poor water wetting (in air or oil) depending on the orientational order and directionality of the $\mathrm{BS}^{-}$groups with respect to the surface plane. This configurational effect should be a better framework to interpret the wetting behavior of a charged surface according to the molecular nature of surface ionic groups. We hope it will stimulate studies to revisit experimental and theoretical molecular design of surface wetting, ${ }^{[17]}$ and that research will focus more on the molecular features of the substituents next to the ionic centers of surface groups, features such such as size, shape, flexibility, and spatial arrangement with regard to the ionic centers rather than simply on the hydration strength of the ionic centers alone. 
Further, the lessons learnt from correlation of the surface wetting behavior of PEMs, obtained by LbL deposition, with their surface local structures will shed light on the study of the PEM internal structures, which is still under debate. Thanks to the operational simplicity and versatility of LbL deposition, our success in using (PDDA/PSS) ${ }_{4}$-coated meshes for efficient oil-water separation also endorses the technical significance of PEMs in self-cleaning applications.

Keywords: interfaces - oil-water separation . photoelectronspectroscopy · structure elucidation . surface chemistry

How to cite: Angew. Chem. Int. Ed. 2015, 54, 4851-4856 Angew. Chem. 2015, 127, 4933-4938

[1] a) J. Israelachvili, H. Wennerstrom, Nature 1996, 379, 219-225; b) U. Raviv, S. Giasson, N. Kampf, J.-F. Gohy, R. Jerome, J. Klein, Nature 2003, 425, 163-165.

[2] a) K. D. Collins, Biophys. J. 1997, 72, 65-76; b) W. Kunz, P. Lo Nostro, B. W. Ninham, Curr. Opin. Colloid Interface Sci. 2002, 7, $81-91$; c) L. B. Sagle, K. Cimatu, V. A. Litosh, Y. Liu, S. C. Flores, X. Chen, B. Yu, P. S. Cremer, J. Am. Chem. Soc. 2011, 133, 18707-18712; d) R. Scheu, Y. Chen, H. B. de Aguiar, B. M. Rankin, D. Ben-Amotz, S. Roke, J. Am. Chem. Soc. 2014 136, 2040-2047.

[3] N. Giovambattista, P. G. Debenedetti, P. J. Rossky, J. Phys. Chem. B 2007, 111, 9581-9587.

[4] M. Ramiasa, J. Ralston, R. Fetzer, R. Sedev, D. M. Fopp-Spori, C. Morhard, C. Pacholski, J. P. Spatz, J. Am. Chem. Soc. 2013, $135,7159-7171$.

[5] D. N. Rao, Colloids Surf. A 2002, 206, 203-216.

[6] a) M. Liu, S. Wang, Z. Wei, Y. Song, L. Jiang, Adv. Mater. 2009, 21, 665-669; b) Y. C. Jung, B. Bhushan, Langmuir 2009, 25, $14165-14173$

[7] a) Multilayer Thin Films (Eds.: G. Decher, J. Schlenoff), WileyVCH, Weinheim, 2003; b) J. F. Quinn, A. P. R. Johnston, G. K. Such, A. N. Zelikin, F. Caruso, Chem. Soc. Rev. 2007, 36, $707-$ 718; c) Z. Y. Tang, Y. Wang, P. Podsiadlo, N. A. Kotov, $A d v$. Mater. 2006, 18, 3203 -3224; d) J. Sun, T. Wu, Y. Sun, Z. Wang, X Zhang, J. Shen, W. Cao, Chem. Commun. 1998, 1853-1854.
[8] R. A. Ghostine, M. Z. Markarian, J. B. Schlenoff, J. Am. Chem. Soc. 2013, 135, 7636-7646.

[9] Note that (PDDA/PSS $)_{55}$ and (PDDA/PSS $)_{60}$ were produced by LbL deposition in $0.50 \mathrm{M} \mathrm{NaCl}$, (PDDA/PSS) 9.5 and (PDDA/ PSS $)_{10.0}$ PEMs in $0.25 \mathrm{M} \mathrm{NaCl}$ and (PDDA/PSS) $)_{14.5}$ and (PDDA/ PSS) ${ }_{15.0}$ PEMs in $0.10 \mathrm{M} \mathrm{NaCl}$, the thickness and surface roughness of which were comparable to that of (PDDA/PSS) $)_{3.5}$ and $(\mathrm{PDDA} / \mathrm{PSS})_{4.0}$ PEMs.

[10] Note that using the experimental $\theta_{\text {w/a }}$ value $\left(52^{\circ}\right)$ of the (PDDA/ PSS $)_{4}$ PEMs obtained after 72 hours of aging at $60^{\circ} \mathrm{C}$, the $\theta_{\mathrm{o} / \mathrm{w}}$ and $\theta_{\mathrm{w} / \mathrm{o}}$ values were calculated at $112^{\circ}$ and $68^{\circ}$, respectively, by using Equations (1) and (2), and they were still significantly smaller than experimental values.

[11] Z. Chen, Prog. Polym. Sci. 2010, 35, 1376-1402.

[12] Note that the $\mathrm{CH}_{2}$ moieties of the PDDA backbone should have a small contribution to the $\mathrm{CH}_{2}$ stretching vibration signal as they may be either shielded by the pyrrolidine ring of the $\mathrm{QA}^{+}$ groups or randomly oriented within the surface plane (Figure 4).

[13] a) F. Zhang, Z. Ali, F. Amin, A. Feltz, M. Oheim, W. J. Parak, ChemPhysChem 2010, 11, 730-735; b) A. Riedinger, F. Zhang, F. Dommershausen, C. Röcker, S. Brandholt, G. U. Nienhaus, U. Koert, W. J. Parak, Small 2010, 6, 2590-2597.

[14] The use of different electrolyte and polyelectrolytes for LbL deposition can yield PEMs with different surface morphology, surface charge, and internal structure (Ref [15]). To avoid that technical issue, new electrolytes and polyelelytrolytes were used only for growth of the outmost layers rather than the entire PEMs. (PDDA/PSS) $)_{3.0}$ and (PDDA/PSS) $)_{3.5}$ PEMs, obtained in $1.0 \mathrm{M} \mathrm{NaCl}$, were used for deposition of polycations and polyanions in $1.0 \mathrm{~m}$ salt, respectively, so the resulting PEMs should be comparable to (PDDA/PSS) $)_{3.5}$ and (PDDA/PSS $)_{4.0}$ PEMs obtained in $1.0 \mathrm{M} \mathrm{NaCl}$ in terms of the film thickness and surface morphology and charge.

[15] R. von Klitzing, Phys. Chem. Chem. Phys. 2006, 8, 5012-5033.

[16] T. Serizawa, S. Hashiguchi, M. Akashi, Langmuir 1999, 15, $5363-5368$

[17] a) A. Ulman, Chem. Rev. 1996, 96, 1533-1554; b) A. Y. Fadeev, T. J. McCarthy, Langmuir 1999, 15, 3759-3766.

Received: December 14, 2014

Revised: January 21, 2015

Published online: February 20, 2015 